\title{
Incorporation of System Resolution Compensation (RC) in the Ordered-Subset Transmission (OSTR) Algorithm for Transmission Imaging in SPECT
}

\author{
Bing Feng* , Jeffrey A. Fessler, and Michael A. King
}

\begin{abstract}
In order to reconstruct attenuation maps with improved spatial resolution and quantitative accuracy, we developed an approximate method of incorporating system resolution compensation (RC) in the ordered-subset transmission (OSTR) algorithm for transmission reconstruction. Our method approximately models the blur caused by the finite intrinsic detector resolution, the nonideal source collimation and detector collimation. We derived the formulation using the optimization transfer principle as in the derivation of the OSTR algorithm. The formulation includes one forward-blur step and one back-blur step, which do not severely slow down reconstruction. The formulation could be applicable to various transmission geometries, such as point-source, line-source, and sheet-source systems. Through computer simulations of the MCAT phantom and transmission measurements of the air-filled Data Spectrum Deluxe single photo emission computed tomography (SPECT) Phantom on a system which employed a cone-beam geometry and a system which employed a scanning-line-source geometry, we showed that incorporation of RC increased spatial resolution and improved the quantitative accuracy of reconstruction. In simulation studies, attenuation maps reconstructed with $\mathrm{RC}$ correction improved the quantitative accuracy of emission reconstruction.
\end{abstract}

Index Terms-Ordered-subset transmission (OSTR), resolution compensation, transmission imaging.

\section{INTRODUCTION}

I $\mathrm{N}$ single photo emission computed tomography (SPECT) attenuation and finite distance-dependent resolution of the imaging system are two sources of image degradation [1]-[4]. High-quality patient-specific attenuation maps are needed to perform accurate attenuation compensation (AC) [5], [6]. Attenuation maps are normally obtained from transmission acquisition using various transmission sources (Ba-133, Tc-99 $\mathrm{m}, \mathrm{Gd}-153$, etc.) and imaging geometries (point source, line source, sheet source, etc.) [7]-[14]. The finite intrinsic spatial

Manuscript received February 13, 2006; revised April 11, 2006. This work was supported by the National Heart, Lung, and Blood Institute under Grant HL50349. The contents are solely the responsibility of the authors and do not necessarily represent the official views of the National Institutes of Health. Asterisk indicates corresponding author.

*B. Feng is with the Department of Radiology, University of Massachusetts Medical School, Worcester, MA 01655 USA (e-mail: bing.feng@umassmed. edu).

M. A. King is with the Department of Radiology, University of Massachusetts Medical School, Worcester, MA 01655 USA (e-mail: michael.king@umassmed.edu).

J. A. Fessler is with Electrical Engineering and Computer Science, University of Michigan, Ann Arbor, MI 48109 USA (e-mail: fessler@eecs.umich.edu).

Digital Object Identifier 10.1109/TMI.2006.876151 resolution of the camera and nonideal photon collimation cause an imprecision in the detected location and assumed origin of the transmission photons, which results in the blurred attenuation maps [15], [16]. Herein, when we speak of system resolution we will be referring to the combination of the intrinsic resolution of the $\mathrm{NaI}(\mathrm{Tl})$ camera, and geometric resolution provided by collimation. For a point source transmission system, Manglos et al. [17] showed that the spatial resolution of the system is close to the intrinsic camera resolution. For extended sheet source transmission system, Cao and Tsui [18] showed that the spatial resolution is further decreased by the nonideal source and camera collimation. For line-source [7], [15] or scanning line-source [12], [19] transmission systems, the spatial resolution can be different between the transverse and axial directions, since in one direction the position of the source is well-defined or the source is well-collimated via electronic collimation and in the other direction it should be treated as the extended source [20] since typically no added source collimation is provided along the line. As the finite resolution of the imaging system causes image degradation in emission reconstruction, it also causes degradation of attenuation maps obtained from transmission reconstructions [15], [16].

Ollinger et al. [21] developed a method of modeling camera intrinsic resolution in the transmission expectation-maximization (EM) algorithm [22]. In their work, a static convolution kernel was utilized to describe the intrinsic resolution. No modeling of the collimation was included in their work. An extended transmission source could be divided into small elements and modeled as overlapping beams to model source and camera collimation [14]. The reconstruction could be performed with the transmission algorithm for overlapping beams [13]. One concern for this approach is the computation cost. The reconstruction time is approximately proportional to the number of overlapping sources. Thus modeling a sheet-source system as 1000 or more overlapping point-sources would mean three or more orders longer reconstruction time compared with the case of no resolution compensation. As an alternative, we extended Ollinger's approach to approximate both the intrinsic camera blur and the blur due to collimation by a static convolution kernel, which describes the average blur of the object in a transmission system with an extended transmission source. We still trace photons along the well-defined path(s) as in the ideal case, but model the blur along each path on average as a static (independent of image space indexes) convolution kernel. Thereafter, we call the convolution kernel the system-blur-function (SBF). The approximation of using the average blur enables the 
use of the system matrix as for an ideal system with perfect spatial resolution. This alleviates the necessity of using the overlapping beams and tracing all possible photon paths, thus greatly reducing the cost of computation. In Section III-B, we will show how to estimate the SBF for a transmission system with sheet source and parallel-hole collimation. Extension to other geometries is also possible. We would like to emphasize that though a static kernel is utilized, this static kernel could be adjusted by the average distance of the object from the camera (or the radius of rotation employed). For transmission systems with point sources, the blur is mainly caused by the camera intrinsic resolution [17]. Thus, the SBF is the blurring kernel that describes the intrinsic camera resolution.

Erdoğan and Fessler [23] proposed a monotonic transmission reconstruction algorithm called the "separable paraboloidal surrogates" (SPS) algorithm, which models Poisson statistics in transmission imaging and can be easily incorporated with a piece-wise smoothness prior. The ordered-subset transmission (OSTR) algorithm [24] is the ordered-subset version of SPS which accelerates efficiently the reconstruction. Herein, we incorporate system resolution compensation (RC) into the OSTR algorithm. Similar to the derivation of the SPS and OSTR algorithms, we adopted the optimization transfer principle [25], [26], which transfers the original optimization task to another easier optimization task or series of easier tasks. We derived the formulation for RC in the frame of the SPS algorithm and adapted it to the OSTR algorithm. It turned out that incorporation of the $\mathrm{RC}$ requires only minor modification to the OSTR algorithm.

\section{METHODS}

In transmission imaging, the measurement model is

$$
y_{i} \sim \operatorname{Poisson}\left\{\bar{y}_{i}\right\}
$$

where $i=1, \ldots, n_{d}$ denotes the detector index. The measurement means for an imaging system with ideal spatial resolution are

$$
\bar{y}_{i}^{0}(\mathbf{x})=b_{i} e^{-[\mathbf{A x}]_{i}}+r_{i}
$$

where $\mathbf{x}=\left[x_{1}, \ldots, x_{j}, \ldots, x_{n_{p}}\right]^{T}$ is a vector describing the three-dimensional (3-D) distribution of the linear attenuation coefficient, $n_{p}$ is the number of voxels, $\mathbf{A}=\left\{a_{i j}\right\} \in \Re^{n_{d} \times n_{p}}$ denotes the system matrix for the transmission system, $\bar{y}_{i}^{0}, b_{i}$, and $r_{i}$ are the ideal counts, flood, and average background (i.e., scatter or crosstalk) at $i$ th detector bin, respectively. For a realistic imaging system with system-blur-function $G=\left\{g_{i m}\right\} \in$ $\Re^{n_{d} \times n_{d}}$, where $g_{i m}$ denotes the detection probability of $\bar{y}_{m}^{0}$ in $i$ th detector due to the average blur along the photon path associated with $m$ th detector bin, the measurement means for a realistic imaging system are

$$
\begin{aligned}
\bar{y}_{i}(\mathbf{x}) & =\sum_{m=1}^{n_{d}} g_{i m} \bar{y}_{m}^{0}=\sum_{m=1}^{n_{d}} g_{i m}\left(b_{m} e^{-[\mathbf{A} \mathbf{x}]_{m}}+r_{m}\right) \\
& =\sum_{m=1}^{n_{d}} g_{i m} b_{m} e^{-[\mathbf{A} \mathbf{x}]_{m}}+\sum_{m=1}^{n_{d}} g_{i m} r_{m},
\end{aligned}
$$

where $g_{i m} \neq 0$ only for rays within the same projection view, since there is no blurring from one projection view into another. If we define $b_{i m} \equiv g_{i m} b_{m}$, and $R_{i} \equiv \sum_{m=1}^{n_{d}} g_{i m} r_{m}$, we have

$$
\bar{y}_{i}(\mathbf{x})=\sum_{m=1}^{n_{d}} b_{i m} e^{-[\mathbf{A} \mathbf{x}]_{m}}+R_{i}
$$

The negative log-likelihood is

$$
\psi(\mathbf{x})=\sum_{n=1}^{n_{d}} \kappa\left(y_{i}, \bar{y}_{i}(\mathbf{x})\right)
$$

where the KL divergence defined as $\kappa(u, v) \equiv u \log (u / v)-u+$ $v$. Thus, the gradient can be obtained according to the following:

$$
\begin{aligned}
\frac{\partial \psi(\mathbf{x})}{\partial x_{j}}= & \sum_{i=1}^{n_{d}}\left(1-\frac{y_{i}}{\bar{y}_{i}(\mathbf{x})}\right) \frac{\partial \bar{y}_{i}(\mathbf{x})}{\partial x_{j}} \\
= & \sum_{i=1}^{n_{d}}\left[\left(1-\frac{y_{i}}{\bar{y}_{i}(\mathbf{x})}\right) \sum_{m=1}^{n_{d}} b_{i m} e^{-[\mathbf{A x}]_{m}}\left(-a_{m j}\right)\right] \\
= & \sum_{m=1}^{n_{d}}\left[\left(-a_{m j}\right) b_{m} e^{-[\mathbf{A x}]_{m}}\right. \\
& \times \sum_{i=1}^{n_{d}}\left(1-\frac{y_{i}}{\bar{y}_{i}(\mathbf{x})}\right) g_{i m} .
\end{aligned}
$$

Instead minimizing $\psi(\mathbf{x})$ directly, we tried to construct the separable surrogate function of $\psi(\mathbf{x})$ [23], [24]. By minimizing or decreasing the surrogate function at each iteration, the optimization transfer principle will guarantee a monotonic decrease of $\psi(\mathbf{x})$ at each iteration and convergence to at least a local minimum [23]. From (3) and (4), we have

$$
\psi(\mathbf{x})=\sum_{i=1}^{n_{d}} \kappa\left(y_{i}, \sum_{m=1}^{n_{d}} b_{i m} e^{-[A x]_{m}}+R_{i}\right) .
$$

To simplify the notation, we define $u_{i m}(\mathbf{x}) \equiv b_{i m} e^{-[\mathbf{A x}]_{m}}+$ $r_{i m}$, and $r_{i m} \equiv R_{i} / n_{d}$, then we have

$$
\begin{aligned}
\psi(x) & =\sum_{i=1}^{n_{d}} \kappa\left(y_{i}, \sum_{m=1}^{n_{d}} u_{i m}(\mathbf{x})\right) \\
& =\sum_{i=1}^{n_{d}} \kappa\left(y_{i}, \sum_{m=1}^{n_{d}}\left(\frac{u_{i m}^{(n)}}{\bar{y}_{i}^{(n)}}\right) u_{i m}(\mathbf{x}) \frac{\bar{y}_{i}^{(n)}}{u_{i m}^{(n)}}\right)
\end{aligned}
$$

where $u_{i m}^{(n)} \equiv u_{i m}\left(\mathbf{x}^{(n)}\right), y_{i}^{(n)} \equiv y_{i}\left(\mathbf{x}^{(n)}\right)$, and $\mathbf{x}^{(n)}$ is the linear attenuation coefficient vector at the $n^{\text {th }}$ iteration. Since $u_{i m}^{(n)} / \bar{y}_{i}^{(n)}$ is nonnegative, $\sum_{m=1}^{n_{d}}\left(u_{i m}^{(n)} / \bar{y}_{i}^{(n)}\right)=1$, and since $\kappa(u, v)=\kappa_{u}(v)$ is convex for $v$, we applied De Pierro's multiplicative trick [25], and generated the following surrogate function:

$$
\varphi_{1}^{(n)}(\mathbf{x}) \equiv \sum_{i=1}^{n_{d}} \sum_{m=1}^{n_{d}} \frac{u_{i m}^{(n)}}{\bar{y}_{i}^{(n)}} \kappa\left(y_{i}, u_{i m}(\mathbf{x}) \frac{\bar{y}_{i}^{(n)}}{u_{i m}^{(n)}}\right) \geq \psi(\mathbf{x})
$$


Using the scaling property $\alpha \kappa(u, v / \alpha)=\kappa(\alpha u, v)$, we have

$$
\begin{aligned}
\varphi_{1}^{(n)}(\mathbf{x}) & =\sum_{i=1}^{n_{d}} \sum_{m=1}^{n_{d}} \kappa\left(\frac{u_{i m}^{(n)}}{\bar{y}_{i}^{(n)}} y_{i}, u_{i m}(\mathbf{x})\right) \\
& =\sum_{i=1}^{n_{d}} \sum_{m=1}^{n_{d}} \kappa\left(y_{i m}^{(n)}, u_{i m}(\mathbf{x})\right) \\
& =\sum_{i=1}^{n_{d}} \sum_{m=1}^{n_{d}} h\left([\mathbf{A x}]_{m} ; y_{i m}^{(n)}, b_{i m}, r_{i m}\right)
\end{aligned}
$$

where $y_{i m}^{(n)} \equiv u_{i m}^{(n)} / \bar{y}_{i}^{(n)} y_{i}$, and $h(t ; y, b, r) \equiv \kappa\left(y, b e^{-t}+r\right)$. The surrogate function for $h(t ; y, b, r)$ [23] is

$$
\begin{aligned}
q(t ; y, b, r, s) \equiv & h(s ; y, b, r)+\dot{h}(s ; y, b, r)(t-s) \\
& +\widetilde{c}(s ; y, b, r) \frac{1}{2}(t-s)^{2} \\
\geq & h(t ; y, b, r)
\end{aligned}
$$

for $\forall s \in \Re$, where $\dot{h}(s ; y, b, r)=(\partial / \partial t) h(t ; y, b, r)$, and $\widetilde{c}(s ; y, b, r)$ is defined in [23]. Substituting (10) into (9), we have the following inequality:

$$
\begin{aligned}
\varphi_{1}^{(n)}(\mathbf{x}) \leq & \sum_{i=1}^{n_{d}} \sum_{m=1}^{n_{d}} q\left([\mathbf{A} \mathbf{x}]_{m} ; y_{i m}^{(n)}, b_{i m}, r_{i m},\left[\mathbf{A} \mathbf{x}^{(n)}\right]_{m}\right) \\
\equiv & \varphi_{2}^{(n)}(\mathbf{x}) \\
= & \sum_{i=1}^{n_{d}} \sum_{m=1}^{n_{d}} h\left(\left[\mathbf{A} \mathbf{x}^{(n)}\right]_{m} ; y_{i m}^{(n)}, b_{i m}, r_{i m}\right) \\
& +\sum_{i=1}^{n_{d}} \sum_{m=1}^{n_{d}} \dot{h}\left(\left[\mathbf{A} \mathbf{x}^{(n)}\right]_{m} ; y_{i m}^{(n)}, b_{i m}, r_{i m}\right) \\
& \times\left([\mathbf{A x}]_{m}-\left[\mathbf{A} \mathbf{x}^{(n)}\right]_{m}\right) \\
& +\sum_{m=1}^{n_{d}} \frac{1}{2}\left([\mathbf{A x}]_{m}-\left[\mathbf{A} \mathbf{x}^{(n)}\right]_{m}\right)^{2} \widetilde{\widetilde{c}}_{m}^{(n)}
\end{aligned}
$$

where $\widetilde{\widetilde{c}}_{m}^{(n)} \equiv \sum_{i=1}^{n_{d}} \widetilde{c}_{i m}^{(n)}$, and $\widetilde{c}_{i m}^{(n)} \equiv$ $\sum_{i=1}^{n_{d}} \widetilde{c}\left(\left[\mathbf{A x} \mathbf{x}^{(n)}\right]_{m} ; y_{i m}^{(n)}, b_{i m}, r_{i m}\right)$.

Further, we can rewrite $\varphi_{2}^{(n)}(\mathbf{x})$ as

$$
\begin{aligned}
\varphi_{2}^{(n)}(\mathbf{x}) & =\psi\left(\mathbf{x}^{(n)}\right)+\left(\nabla \psi\left(\mathbf{x}^{(n)}\right)\right)^{T}\left(\mathbf{x}-\mathbf{x}^{(n)}\right) \\
+ & \frac{1}{2}\left(\mathbf{x}-\mathbf{x}^{(n)}\right)^{T} \mathbf{A}^{T} \operatorname{diag}\left\{\widetilde{\widetilde{c}}_{m}^{(n)}\right\} \mathbf{A}\left(\mathbf{x}-\mathbf{x}^{(n)}\right)
\end{aligned}
$$

where $\left(\nabla \psi\left(\mathbf{x}^{(n)}\right)\right)^{T},\left(\mathbf{x}-\mathbf{x}^{(n)}\right)^{T}$, and $\mathbf{A}^{T}$ are transposes of $\nabla \psi\left(\mathbf{x}^{(n)}\right),\left(\mathbf{x}-\mathbf{x}^{(n)}\right)$, and $\mathbf{A}$, respectively, and $\operatorname{diag}\left\{\widetilde{\widetilde{c}}_{m}^{(n)}\right\} \equiv$ $\left\{\widetilde{c}_{m}^{(n)} \delta_{m j}\right\} \in \Re^{n_{d} \times n_{d}}$, where $\delta_{m j}=1$ for $m=j$, otherwise $\delta_{m j}=0$. Using the inequality from [23] $\mathbf{B}^{T} \operatorname{diag}\left\{w_{i}\right\} \mathbf{B} \leq$ $\operatorname{diag}\left\{\sum_{i=1}^{n_{d}} w_{i} b_{i j} b_{i}\right\}$, for any matrix $\mathbf{B}=\left\{b_{i j}\right\} \in \Re^{n_{d} \times n_{p}}$, where $b_{i} \equiv \sum_{j=1}^{n_{p}}\left|b_{i j}\right|$, we have

$$
\mathbf{A}^{T} \operatorname{diag}\left\{\widetilde{c}_{m}^{(n)}\right\} \mathbf{A} \leq \operatorname{diag}\left\{\sum_{m=1}^{n_{d}} \widetilde{\widetilde{c}}_{m}^{(n)} a_{m j} a_{m}\right\} \equiv \operatorname{diag}\left\{d_{j}^{(n)}\right\}
$$

where $a_{m} \equiv \sum_{j=1}^{n_{p}}\left|a_{m j}\right|=\sum_{j=1}^{n_{p}} a_{m j}$, and $d_{j}^{(n)} \equiv$ $\sum_{m=1}^{n_{d}} \widetilde{\widetilde{c}}_{m}^{(n)} a_{m j} a_{m}$. Substituting (13) into (12), we obtained a separable surrogate function

$$
\begin{aligned}
& \varphi_{3}^{(n)}(\mathbf{x})=\psi\left(\mathbf{x}^{(n)}\right)+\left(\nabla \psi\left(\mathbf{x}^{(n)}\right)\right)^{T}\left(\mathbf{x}-\mathbf{x}^{(n)}\right) \\
& +\frac{1}{2}\left(\mathbf{x}-\mathbf{x}^{(n)}\right)^{T} \operatorname{diag}\left\{d_{j}^{(n)}\right\}\left(\mathbf{x}-\mathbf{x}^{(n)}\right) \geq \varphi_{2}^{(n)}(\mathbf{x}) .
\end{aligned}
$$

Minimizing $\varphi_{3}^{(n)}(\mathbf{x})$ and enforcing the nonnegativity constraint, we obtained the $(n+1)^{t h}$ update as

$$
x_{j}^{(n+1)}=\left[x_{j}^{(n)}-\frac{1}{d_{j}^{(n)}} \frac{\partial \psi\left(\mathbf{x}^{(n)}\right)}{\partial x_{j}}\right]_{+}
$$

where the operator $[a]_{+}=a$ for $a>0$, and zero otherwise. From (5), we have the gradient

$$
\begin{aligned}
\frac{\partial \psi\left(\mathbf{x}^{(n)}\right)}{\partial x_{j}}=\sum_{m=1}^{n_{d}}\left[\left(-a_{m j}\right) b_{m} e^{-\left[\mathbf{A} \mathbf{x}^{(n)}\right]_{m}}\right. & \\
& \times \sum_{i=1}^{n_{d}}\left(1-\frac{y_{i}}{\bar{y}_{i}\left(\mathbf{x}^{(n)}\right)}\right) g_{i m} .
\end{aligned}
$$

To calculate the gradient as in (16), one should perform one forward-blur in $\bar{y}_{i}\left(\mathbf{x}^{(n)}\right)=\sum_{m=1}^{n_{d}} g_{i m}\left(b_{m} e^{-\left[\mathbf{A} \mathbf{x}^{(n)}\right]_{m}}+r_{m}\right)$ and one back-blur in $\sum_{i=1}^{n_{d}}\left(1-\left(y_{i} / \bar{y}_{i}\left(\mathbf{x}^{(n)}\right)\right)\right) g_{i m}$. Taking into account $g_{i m} \neq 0$ only for rays within the same projection view, these blurring steps are performed frame-by-frame within projection/backprojection and do not affect severely reconstruction time. Thus, far the monotonicity still holds.

To adapt our method to the OSTR algorithm, we replaced the sum over $m$ in (16) with the sum over subsets of the rays, and approximated $d_{j}^{(n)}$ with $d_{j}$, which is independent of the iteration number thus achieving a fast implementation [24]. From (13), we have

$$
\begin{aligned}
d_{j}^{(n)} & \equiv \sum_{m=1}^{n_{d}} \widetilde{\widetilde{c}}_{m}^{(n)} a_{m j} a_{m}=\sum_{m=1}^{n_{d}} a_{m j} a_{m} \sum_{i=1}^{n_{d}} \widetilde{c}_{i m}^{(n)} \\
& =\sum_{m=1}^{n_{d}} a_{m j} a_{m} \sum_{i=1}^{n_{d}} \widetilde{c}\left(\left[\mathbf{A} \mathbf{x}^{(n)}\right]_{m} ; y_{i m}^{(n)}, b_{i m}, r_{i m}\right) \\
& \approx \sum_{m=1}^{n_{d}} a_{m j} a_{m} \sum_{i=1}^{n_{d}} y_{i m}^{(n)} \\
& =\sum_{m=1}^{n_{d}} a_{m j} a_{m} \sum_{i=1}^{n_{d}} \frac{u_{i m}^{(n)}}{\bar{y}_{i}^{(n)}} y_{i} .
\end{aligned}
$$


Since the blur caused by the finite detector resolution is small, we approximated $u_{i m}^{(n)} / \bar{y}_{i}^{(n)} \approx \delta_{i m}$, where $\delta_{i m}=1$ if $i=m$, otherwise $\delta_{i m}=0$. Making use of (17), we have

$$
d_{j}^{(n)} \approx \sum_{m=1}^{n_{d}} a_{m j} a_{m} \sum_{i=1}^{n_{d}} y_{i} \delta_{i m}=\sum_{m=1}^{n_{d}} a_{m j} a_{m} y_{m} \equiv d_{j}
$$

Finally, we obtained the formulation for incorporating system resolution compensation in the OSTR algorithm

$$
\begin{aligned}
& x_{j}^{(n+1)} \\
& =\left[x_{j}^{(n)}+\frac{M \sum_{m \in S} a_{m j} b_{m} e^{-\left[\mathbf{A} \mathbf{x}^{(n)}\right]_{m} \sum_{i=1}^{n_{d}}\left(1-\frac{y_{i}}{\bar{y}_{i}\left(\mathbf{x}^{(n)}\right)}\right) g_{i m}}}{d_{j}}\right]_{+}
\end{aligned}
$$

where $S$ is one of $M$ subsets of the rays. For simplicity we omitted the usual terms for regularization [23] in (19). Taking into account $g_{i m} \neq 0$ only for rays within the same projection view, we rewrite (19) explicitly as

$$
\begin{aligned}
& x_{j}^{(n+1)} \\
& =\left[x_{j}^{(n)}+\frac{M \sum_{m \in S} a_{m j} b_{m} e^{-\left[\mathbf{A} \mathbf{x}^{(n)}\right]_{m} \sum_{i \in S}\left(1-\frac{y_{i}}{\bar{y}_{i}\left(\mathbf{x}^{(n)}\right)}\right) g_{i m}}}{d_{j}}\right]_{+}
\end{aligned}
$$

where $\bar{y}_{i}\left(\mathbf{x}^{(n)}\right)=\sum_{m^{\prime} \in S}\left(b_{m^{\prime}} e^{\left.-\left[\mathbf{A} \mathbf{x}^{(n)}\right]_{m^{\prime}}+r_{m^{\prime}}\right)}\right.$.

\section{COMPUTER SimulationS AND TRANSMISSION MEASUREMENTS}

We evaluated our method for two transmission imaging geometries. The first is a modified Beacon system [10] on an IRIX SPECT system (Philips Medical Systems, Cleveland, $\mathrm{OH}$ ), which employs cone-beam imaging of medium-energy photons from point sources that penetrate the septa of opposed low-energy parallel-hole collimators. The second is a scanning-line-source system on a PRISM 2000 (Philips Medical Systems, Cleveland, OH). Based on each system, computer simulations were performed with MCAT phantom [27], with clinically equivalent noise levels. These levels were determined from clinical transmission scans acquired with each of these systems. To evaluate our approach with more realistic transmission data, transmission measurements of the air-filled Data Spectrum Deluxe SPECT Phantom were also performed on each system.

To investigate the impact of modeling system resolution in transmission reconstruction on attenuation compensation with emission reconstruction, we generated transmission and emission projection data of the MCAT phantom. We then performed attenuation compensation as part of iterative reconstruction of simulated MCAT emission projections. We compared the emission slices resulting from use of the true MCAT attenuation map, and the attenuation maps reconstructed with and without $\mathrm{RC}$ incorporated in transmission reconstruction. Since we expected the most difference to occur when using RC in transmission reconstruction for the case of the scanning-line-source system, we evaluated attenuation compensation with attenuation maps simulated solely for the scanning-line-source system.

\section{A. Point Source With Cone-Beam Geometry (Modified $B E A C O N)$}

We modified the BEACON transmission system in our clinic and turned the original scanning point source system (fan-beam geometry) into a stationary point source system (cone-beam geometry) [10]. To cover a wider axial extent, each of the two point sources irradiating opposite heads are axially separated by $7 \mathrm{~cm}$. Using such a cone-beam geometry, fast sequential transmission scan can be performed in $2 \mathrm{~min}$. The axial coverage of the modified BEACON is about $15.4 \mathrm{~cm}$, which is adequate for cardiac imaging. If the size of the point source is negligible, the spatial resolution of the modified BEACON system at camera plane is equal to the intrinsic resolution of the gamma camera, which is a sigma of about $0.2 \mathrm{~cm}\left(\sigma_{i}=0.2 \mathrm{~cm}\right)$ for the $356-\mathrm{keV}$ photons of Ba-133, as imaged by the 1.9-cm-thick crystals of the system.

A ray-driven numerical projector and a flood measured on the modified BEACON system were utilized to simulate the transmission projection data of the MCAT attenuation map. The projection data contain 120 views of $128 \times 128$ images for each head, covering $360^{\circ}$. The radius-of-rotation (ROR) was $30 \mathrm{~cm}$. The pixel size was $0.416 \mathrm{~cm}$. The projection data were convolved with a two-dimensional (2-D) Gaussian with a $0.2-\mathrm{cm}$ sigma, to simulate blurring due to the intrinsic resolution of the camera. Then Poisson noise was added. The total number of counts on each head was about 1.5 million, simulating a two-minute acquisition. The projection data were reconstructed by 50 iterations of the OSTR algorithm, with and without RC. The same 3-D edge-preserving smoothness prior [28] was used in both reconstructions. No post smoothing was applied. The root-mean-square error (RMSE) in a region of interest (ROI) was calculated to evaluate the quantitative accuracy of reconstructions, according to the following formula:

$$
\text { RMSE }=\sqrt{\frac{\sum_{i \in \mathrm{ROI}}\left(x_{i}-x_{i}^{\text {true }}\right)^{2}}{N}}
$$

where the ROI contains the entire 3-D volume of the attenuation map within the axial coverage, $N$ is the number of voxels inside the ROI, and $x_{i}$ and $x_{i}^{\text {true }}$ are the reconstructed and true values of the linear attenuation coefficient at voxel $i$, respectively.

In addition, two 30-min transmission acquisitions of an airfilled Data Spectrum Deluxe phantom were performed on the modified BEACON system. The two acquisitions differed from each other by the ROR employed. The ROR was $25 \mathrm{~cm}$ for the first acquisition and $32 \mathrm{~cm}$ for the second acquisition. The $25-\mathrm{cm}$ ROR was selected as the shortest which might be used with a patient, and the 32-cm ROR was selected as the longest. The combination allowed use to investigate changes which might occur over this range of RORs. Each acquisition was reconstructed with 50 iterations of the OSTR algorithm with and without RC. 
The 120 views per head (with two heads, each rotating $360^{\circ}$ ) were divided equally into 30 subsets, with four views per subset.

\section{B. Scanning Line Source With Parallel-Hole Collimated Camera (PRISM 2000)}

On the PRISM 2000 system in our clinic, transmission imaging utilizes a scanning Gd-153 line source. The normal acquisition time for transmission study is about $20 \mathrm{~min}$. The line source scans in the axial direction and the region of the camera face on the head opposed to the line source which is employed to acquire transmission counts scans electronically in synchrony with the line source. As is typical of such systems, no collimation of the line source in the transaxial direction is incorporated into the line-source holder. Thus, in this direction, the line source is an uncollimated extended source. For simplicity, we assumed the spatial resolution was the same in both directions, and could be described by a 2-D Gaussian function [29], [30]. That is, we assumed the geometry was that of using a sheet source with no source collimation and parallel-hole collimation on the camera. This over-estimates the blurring in the axial direction since the scanning line source is axially collimated by an electronically scanning window and source holder. One reason for employing this simplification was that we were only interested in the transaxial resolution in our experiments with the air-filled Data Spectrum Deluxe SPECT Phantom.

In the simulations with MCAT phantom, the geometric response $\left(\sigma_{g}\right)$ was assumed to be $1.2 \mathrm{~cm}$ (sigma) for a $60-\mathrm{cm}$ source-camera distance. A ray-driven numerical projector was used to trace the cone-beam rays from each source bin on measured flood. The flood was scaled to 36 counts per camera bin (with $0.416 \mathrm{~cm} / \mathrm{bin}$ ) on average, which was close to the noise level of transmission scans we acquire clinically with this system. Counts along each cone-beam ray were multiplied by a weight calculated from the 2-D Gaussian $\left(\sigma_{g}=1.2 \mathrm{~cm}\right)$, and were summed over all source bins into projection data (Fig. 1). The projection data were further convolved with a 2-D Gaussian $\left(\sigma_{i}=0.12 \mathrm{~cm}\right)$ to model the blur due to the intrinsic resolution of the camera PRISM 2000 camera for Gd-153 photons. Poisson noise was then added. In this way we obtained transmission projection data which contained 60 views (over $180^{\circ}$ ) of $128 \times 128$ images.

To estimate the system-blur-function for the imaging geometry described above (Fig. 1), we investigate the counts $\left(I_{t}\right)$ arriving at a certain detector bin through an arbitrary ray $L_{t}$ (the thick solid line in Fig. 1). We have $I_{t}=I_{0} e^{-\left(t^{2} / 2 \sigma_{g}^{2}\right)} e^{-\int_{L_{t}} u d l}$. Approximating the attenuation path $L_{t}$ by the dashed line $L_{d}$, and using similarity relation, we obtain $I_{t} \approx I_{0} e^{-\left(t_{a}^{2} / 2 \sigma_{a}^{2}\right)} e^{-\int_{L_{d}} u d l}$, where $t_{a}=(\mathrm{ROR} / D) t$, $\sigma_{a}=(\mathrm{ROR} / D) \sigma_{g}$, ROR is the radius of rotation, $D$ is the distance between the source and the camera. The average blurring due to nonideal collimation can be approximated by a 2-D Gaussian with sigma $=\sigma_{a}$ [31]. The system-blur-function was defined as the average blurring (sigma $=\sqrt{\sigma_{a}^{2}+\sigma_{i}^{2}}, \sigma_{i}$ is the intrinsic resolution) of object, which was equal to the blurring at the camera plane in measuring the object center or center of rotation (COR) for convenience. Calculated from imaging

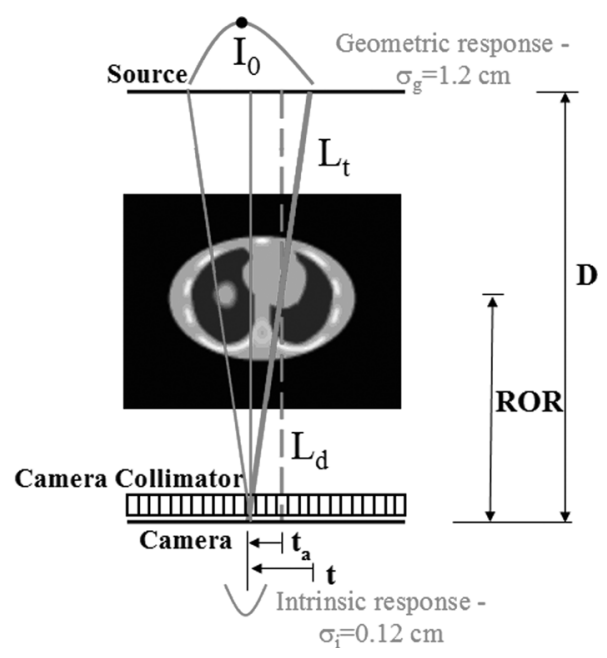

Fig. 1. Transmission system with sheet source and parallel-hole camera collimation is used in simulation. Geometric response due to camera collimation is assumed a 2-D Gaussian. Cone-beam rays in the simulation are plotted in solid lines.

parameters described before, the sigma of the system-blur-function was $0.61 \mathrm{~cm}$, taking into account contributions from both geometric response and intrinsic resolution.

The approximation we made above to estimate SBF normally holds, since the geometric response $\sigma_{g}$ is small when compared with $D$, and the Gaussian decreases rapidly from its central maximum. The more $L_{t}$ deviates from $L_{d}$, the less it contributes to the counts. To have a better understanding of the approximation involved in our simulation, we considered rays $L_{t}$ in Fig. 1 that correspond to the full-width at half-maximum (FWHM) and the full-width at tenth-maximum (FWTM). The tilting angles between $L_{t}$ and $L_{d}$ were $4.2^{\circ}$ and $7.7^{\circ}$, respectively. The separations at the camera plane between $L_{t}$ and $L_{d}$ were 0.71 and 1.3 $\mathrm{cm}$, respectively. At the perimeter of the phantom, this separation were reduced approximately in half, or $0.36 \mathrm{~cm}$ for FWHM and $0.65 \mathrm{~cm}$ for FWTM.

The projection data were reconstructed by 50 iterations of the OSTR algorithm with and without RC. A 3-D edge-preserving smoothness prior [28] was used in both cases. In each case, the data were reconstructed with a range of values (from 1000 to 10000 , with 500 step size) of the scaling factor of the smoothness prior, which controls the tradeoff between the smoothness and resolution. The RMSE within an ROI, which contained the entire MCAT phantom, was calculated for each reconstruction, and the optimal scaling factor of the smoothness prior was chosen, basing on the minimum RMSE. The reconstruction with the optimal smoothness prior corresponded to the best attenuation map we could obtain in each case. The same scaling parameters were applied in latter reconstruction of the acquisitions of the air-filled Data Spectrum Deluxe phantom.

Two 30 minute transmission acquisitions of the air-filled Data Spectrum Deluxe phantom were additionally performed on the PRISM 2000 system. Since the air-filled phantom would not attenuate the Gd-153 line source used clinically enough to make the transmission counting rate similar to that when acquiring clinically, we employed a Gd-153 line-source that had decayed down by approximately four half-lives with the 
air-filled phantom. Thus the transmission counts acquired by a 30-min acquisition were similar to that we obtain in the 20-25-min clinical acquisitions. The two acquisitions differed from each other by the ROR employed: $18.5 \mathrm{~cm}$ in one case and $30 \mathrm{~cm}$ the other. Each acquisition was reconstructed with 50 iterations of the OSTR algorithm with and without RC. The 60 views (covering $180^{\circ}$ ) were divided equally into 15 subsets, with 4 views per subset.

\section{MCAT Phantom Simulations to Study the Impact of System Resolution Compensated Attenuation Maps on Attenuation Compensation During Emission Reconstruction}

We generated a set of emission projection data of the MCAT phantom using a numerical projector which modeled attenuation, distance-dependent system resolution, but no scatter. We simulated a $180^{\circ}$ acquisition with a parallel-hole collimated camera. The projection data consisted of 60 views of $128 \times 128$ bins. The bin size was $0.416 \mathrm{~cm}$. A half-million counts were simulated for the heart which is approximately what we observe in a stress Tc-99 m labeled sestamibi acquisition. Poisson noise was added to the projection data.

We reconstructed the projection data with 10 iterations of the OSEM (15 subsets, four views for each subset). In reconstruction, system resolution and attenuation were modeled in the projector and backprojector. Three attenuation maps were compared: the true attenuation map that matched the simulation, the attenuation map reconstructed without modeling the system resolution, and the attenuation map reconstructed with modeling the system resolution. The latter two attenuation maps were simulated with for a scanning-line-source system (Section III-B). A post Gaussian filter with 1.5 pixel sigma was applied to all reconstructed emission images.

\section{RESULTS}

\section{A. Point Source With Cone-Beam Geometry (Modified $B E A C O N)$}

The results for the reconstruction of the MCAT phantom are shown in Figs. 2 and 3. Since blurring due to the intrinsic resolution is small (sigma $=0.2 \mathrm{~cm}$ in simulation), the difference between reconstructions with $\mathrm{RC}$ and without $\mathrm{RC}$ is also small. The RMSE was slightly smaller in the case with RC $(0.022$ $\left.\mathrm{cm}^{-1}\right)$ than without $\mathrm{RC}\left(0.023 \mathrm{~cm}^{-1}\right)$.

Results from reconstruction of measurements of the air-filled Data Spectrum Deluxe SPECT Phantom are plotted in Fig. 4. $\mathrm{RC}$ slightly increased the resolution of images in that some of the small rods are more clearly seen.

\section{B. Scanning Line Source With Parallel-Hole Collimated Camera (PRISM 2000)}

The transmission projection data of MCAT phantom were simulated for PRISM 2000 system and reconstructed with and without RC. The smoothness prior was optimized in each case to reconstruct images with the minimum RMSE. The minimum RMSE was $0.027 \mathrm{~cm}^{-1}$ without $\mathrm{RC}$, and $0.017 \mathrm{~cm}^{-1}$ with $\mathrm{RC}$. The attenuation map reconstructed with $\mathrm{RC}$ was much smoother than the map without RC. Reconstruction with RC was visually of higher resolution (Fig. 5) and accuracy (Fig. 6).

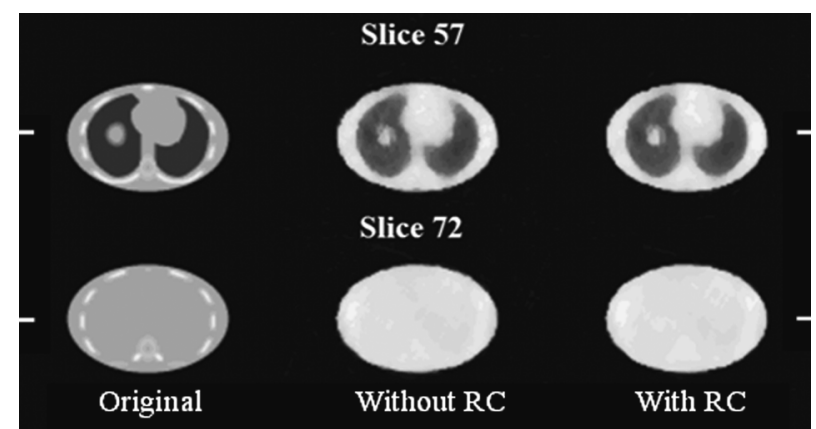

Fig. 2. Reconstruction of the projection data simulated for the modified BEACON system. (Left) Two slices of original MCAT phantom. (Middle) 50 iterations of the OSTR without RC. (Right) 50 iterations of the OSTR with RC. System-blur-function in RC is a 2-D Gaussian with sigma $=0.2 \mathrm{~cm}$. Note that on each slice are shown short bars, along which the profile of attenuation coefficient was obtained for comparison in Fig. 3.

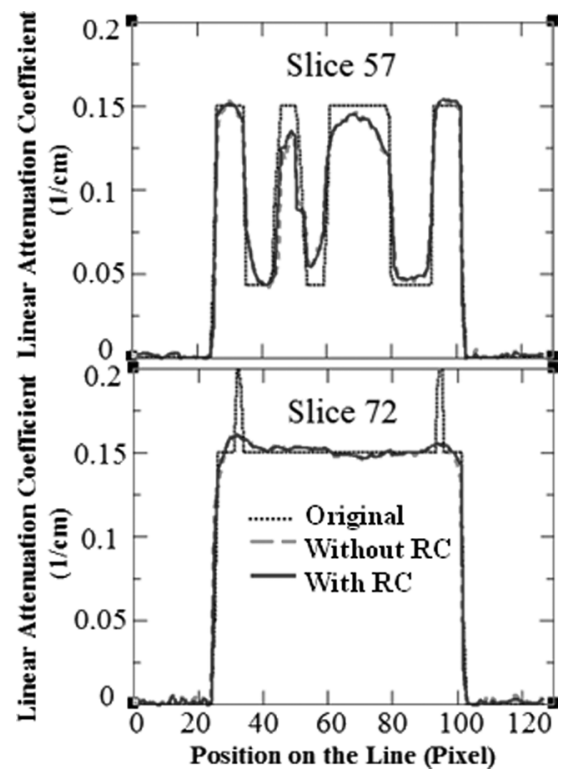

Fig. 3. Profiles of linear attenuation coefficient are plotted through the short bars in Fig. 2. Dotted line is original phantom. Dashed line is without RC. Solid line is with RC.

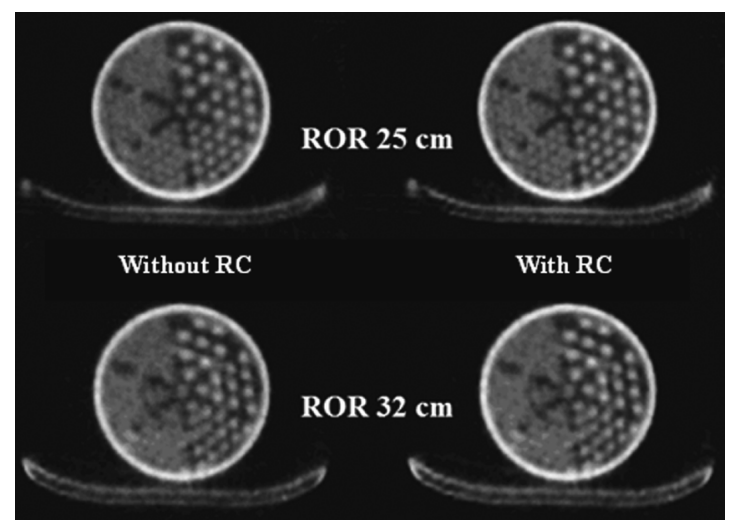

Fig. 4. Reconstruction of transmission measurements $(\mathrm{ROR}=25 \mathrm{~cm}$ and ROR $=30 \mathrm{~cm}$ ) on the modified BEACON system. (Upper left) ROR = $25 \mathrm{~cm}$, without RC. (Upper right) ROR $=25 \mathrm{~cm}$, with RC. (Lower left) $\mathrm{ROR}=32 \mathrm{~cm}$, without RC. (Lower right) $\mathrm{ROR}=32 \mathrm{~cm}$, with RC.

Measurements of the air-filled Data Spectrum Deluxe SPECT Phantom on PRISM 2000 were reconstructed with and without 


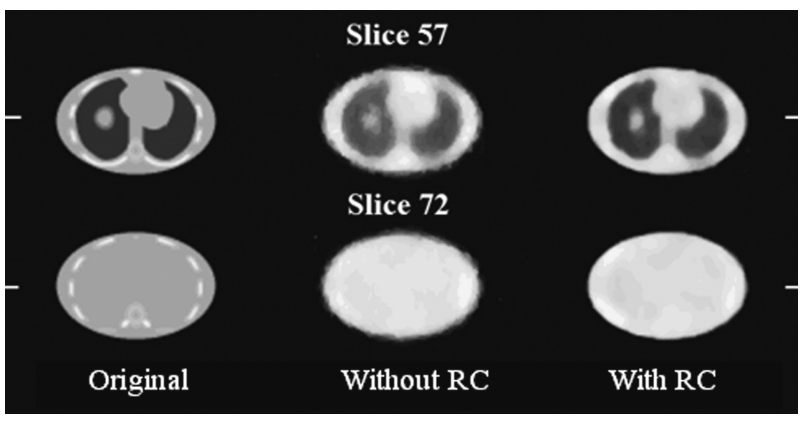

Fig. 5. Reconstruction of the projection data simulated for the PRISM 2000 system. (Left) Two slices of original MCAT phantom. (Middle) 50 iterations of the OSTR without RC. (Right) 50 iterations of the OSTR with RC. Systemblur-function in RC is a 2-D Gaussian with sigma $=0.61 \mathrm{~cm}$. Note that on each slice are shown short bars, along which the profile of attenuation coefficient was obtained for comparison in Fig. 6 .

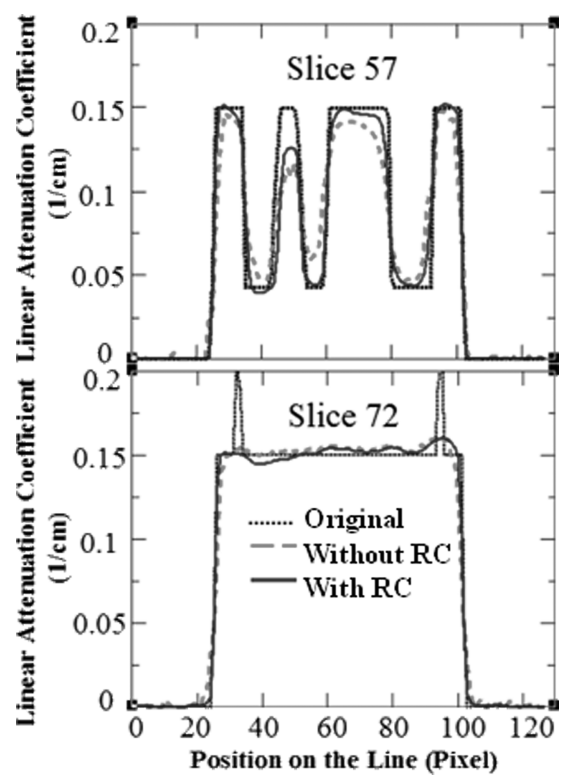

Fig. 6. Profiles of linear attenuation coefficient are plotted through the short bars in Fig. 5. Dotted line is the original phantom. Dashed line is without RC. Solid line is with RC.

RC. In each case, the smoothness prior was the same as for the counterpart in simulations. The results are plotted in Fig. 7. The images reconstructed with RC show better resolution than without RC.

\section{MCAT Phantom Simulations to Study the Impact of the System Resolution Compensated Attenuation Map on the Attenuation Compensation}

The MCAT emission projection data were reconstructed with the true and attenuation maps reconstructed from transmission projection imaging simulating the PRISM 2000 for the cases of with and without RC. A transaxial emission slice of the heart for each case is shown in Fig. 8. Visually no difference could be seen in these images. Using reconstruction with the true attenuation map as the gold standard, the difference (or error) image was calculated for emission reconstruction using attenuation maps reconstructed with and without RC. In maximum error when using the nonresolution compensated attenuation maps

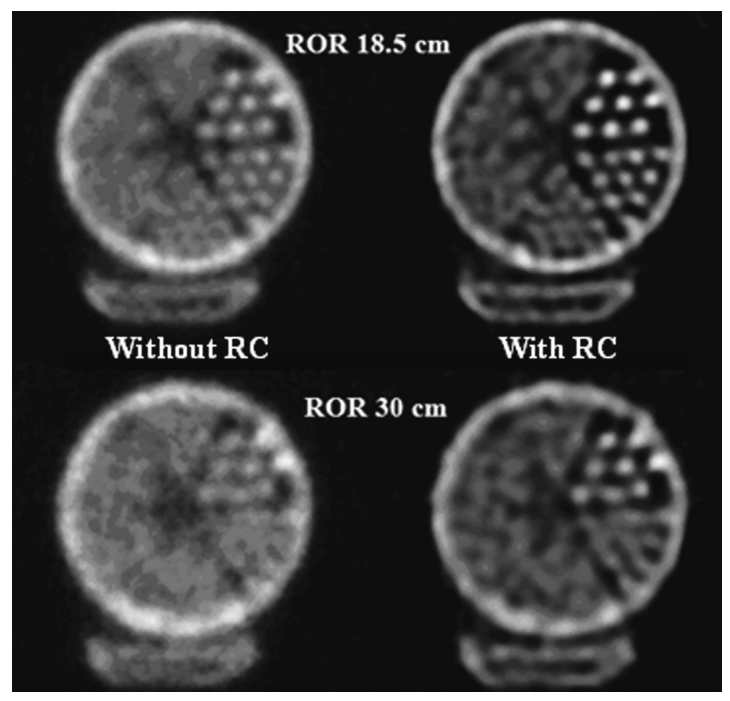

Fig. 7. Reconstruction of transmission measurements $(\mathrm{ROR}=18.5 \mathrm{~cm}$ and ROR $=30 \mathrm{~cm}$ ) on the modified BEACON system. (Upper left) $\mathrm{ROR}=$ $18.5 \mathrm{~cm}$, without RC. (Upper right) ROR $=18.5 \mathrm{~cm}$, with RC (sigma $=$ $0.45 \mathrm{~cm}$ ). (Lower left) ROR $=30 \mathrm{~cm}$, without RC. (Lower right) $\mathrm{ROR}=$ $30 \mathrm{~cm}$, with RC ( sigma $=0.55 \mathrm{~cm})$.

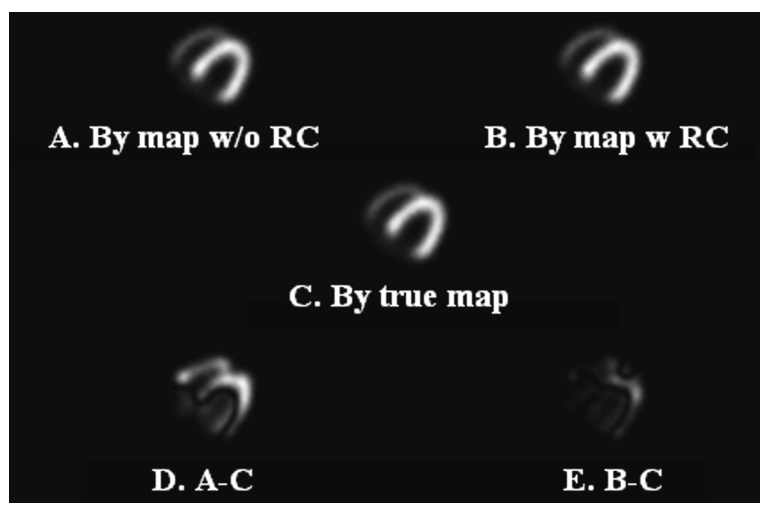

Fig. 8. Attenuation-compensated emission reconstruction of the MCAT phantom simulation. (a) Using the attenuation map reconstructed without system resolution compensation (as shown in the middle column of Fig. 5). (b) Using the attenuation map reconstructed with system resolution compensation (as shown in the right column of Fig. 5). (c) Using the true attenuation map (as shown in the left column of Fig. 5). (d) Absolute difference between A and C. (e) Absolute difference between B and C. (d) and (e) are plotted on the same greyscale.

was about $6 \%$ of the average myocardial counts. This maximum error was reduced to $2 \%$ for the case of the using the attenuation map with RC. The largest difference was found near the apex.

\section{DISCUSSION}

Our method requires two additional smoothing steps, one in the projector, and another in the backprojector. These smoothing steps are performed frame-by-frame and usually with a relatively short convolution kernel. The increase in computation time is a few percent (3\% for the cone-beam case, $5 \%$ for the parallel-beam case) over not including RC. From our experience with overlapping cone-beam transmission imaging [10], the computation time approximately doubles when the number of the point-sources doubles. Thus, by estimation the overlapping beam approach in the one dimensional case will lead to one 
or two orders of magnitude increase in the computation time. To model both axial and transaxial blurring for a scanning line source system, the overlapping-beams approach could increase the computation time by two to three orders.

For a scanning line-source system, our method employs the central-ray approximation for the path of the photon through an attenuating media in the presences of blurring as discussed by Liang, et al. [31]. It could be further improved by using a smoothing kernel determined by the average distance of the object along each ray to the camera. This will require pre-reconstruction and segmentation of the attenuation map. As a result, the smoothing kernel could vary point-by-point on the camera, depending on the average distance of the object to each camera bin.

Only a slight difference was found in the attenuation maps reconstructed with $\mathrm{RC}$ in comparison to those reconstructed without $\mathrm{RC}$ for the case of transmission imaging with a point source. Since this is intrinsically a high-resolution imaging geometry, the lack of a significant difference is to be expected. For the case of transmission imaging with an uncollimated extended source (transaxial slices for an axial scanning-line-source), a significant difference was observed visually and quantitatively in the attenuation maps with use of RC in transmission reconstruction. In transmission imaging for SPECT and positron emission tomography (PET), the attenuation maps are not used for diagnostic purposes. They are used for attenuation compensation. Thus, the best measure of the impact of including $\mathrm{RC}$ in transmission reconstruction is whether the use of RC alters emission slices. It has previously shown that the spatial resolution of the attenuation map should be similar to that of the emission slices to be reconstructed [6]. Our method has been shown to improve the spatial resolution for scanning-line-source systems which employ an uncollimated line-source. When we investigated the difference RC attenuation maps made in emission slices of the MCAT phantom no obvious visual difference was noticed, but the emission slices were quantitatively closer to the emission slices reconstructed with true attenuation map of the MCAT simulation. Thus, we expect our method could improve quantification and in some cases possibly detection accuracy of small lesions near boundaries between organs.

Scatter was not included in our transmission simulations, and the air-filled Data Spectrum phantom has only minimal scatter associated with it. Certainly the presence of scatter in the transmission projections will bias the estimated values of the attenuation coefficients. The OSTR algorithm can perform scatter compensation in reconstruction, if the contribution of scatter to the projection data is known. Herein we concentrated on investigating the use of RC with the OSTR algorithm. In future work, we plan to investigate the use of scatter compensation with the OSTR algorithm.

\section{CONCLUSION}

We developed a method of incorporating compensation for the finite spatial resolution of transmission imaging in the OSTR algorithm. We determined the inclusion of RC can enhance the resolution and accuracy of the reconstructed attenuation maps for scanning-line-source transmission imaging geometries. For other geometries such as scanning point-sources, multiple linesources, and sheet-sources our method is also applicable. The key is to figure out the system-blur-function which describes the average blurring of imaging the object with those systems.

\section{REFERENCES}

[1] M. A. King, B. M. W. Tsui, and T.-S. Pan, "Attenuation compensation for cardiac sigle-photon emission computed tomography imaging: Part 1. Impact of attenuation and methods of estimating attenuation maps," J. Nucl. Cardiol., vol. 2, pp. 513-524, 1995.

[2] S. J. Glick, B. C. Penney, M. A. King, and C. L. Byrne, "Noniterative compensation for the distance-dependent detector response and photon attenuation in SPECT imaging," IEEE Trans. Med. Imag., vol. 13, no. 2, pp. 363-374, Jun. 1994.

[3] G. L. Zeng, G. T. Gullberg, B. M. W. Tsui, and J. A. Terry, "Three-dimensional iterative reconstruction algorithm with attenuation and geometric point response correction," IEEE Trans. Nucl. Sci., vol. 38, no. 2, pp. 693-702, Apr. 1991.

[4] B. M. W. Tsui, H. B. Hu, D. R. Gilland, and G. T. Gullberg, "Implementation of simultaneous attenuation and detector response correction in SPECT," IEEE Trans. Nucl. Sci., vol. 35, no. 1, pp. 778-783, Feb. 1988.

[5] A. Celler, K. L. Dixon, Z. Chang, S. Blinder, J. Powe, and R. Harrop, "Problems created in attenuation-corrected SPECT images by artifacts in attenuation maps: A simulation study," J. Nucl. Med., vol. 46, pp. 335-343, 2005.

[6] B. M. W. Tsui, X. D. Zhao, D. S. Lalush, E. C. Frey, R. E. Johnston, L. S. Bujenovic, and W. H. McCartney, "Pitfalls of attenuation compensation and their remedies in cardiac SPECT," presented at the Soc. Nucl. Med. 41st Annu. Meeting, Orlando, FL, Jun. 5-8, 1994.

[7] G. T. Gullberg, H. T. Morgon, G. L. Zeng, P. E. Christian, E. V. R. Di Bella, C.-H. Tung, P. J. Maniawski, Y.-L. Hsieh, and F. L. Datz, "The design and performance of a simultaneous transmission and emission tomography system," IEEE Trans. Nucl. Sci., vol. 45, no. 3, p. 1676, Jun. 1998.

[8] G. L. Zeng, G. T. Gullberg, and P. E. Christian, "Asymmetric conebeam transmission tomography," IEEE Trans. Nucl. Sci., vol. 48, no. 1, pp. 117-124, Feb. 2002.

[9] D. Gagnon, C. H. Tung, G. L. Zeng, and W. Hawkins, "Design and early testing of a new medium-energy transmission device for attenuation correction in SPECT and PET," in Conf. Rec. 1999 IEEE Nucl. Sci. Symp., Seattle, WA, Oct. 24-30, 1999, pp. 1349-1353.

[10] B. Feng, M. A. King, G. L. Zeng, P. H. Pretorius, P. P. Bruyant, R. Beach, G. Boening, G. Jarkewicz, S. Cochoff, and D. Gagnon, "The estimation of attenuation maps for cardiac-SPECT using cone-beam imaging of high-energy photons through parallel-hole collimators," IEEE Trans. Nucl. Sci., vol. 51, no. 5, pp. 2699-2704, Oct. 2004.

[11] F. J. Beekman, C. Kamphuis, B. F. Hutton, and P. P. van Rijk, "Halffanbeam collimators combined with scanning point sources for simultaneous emission-transmission imaging," J. Nucl. Med., vol. 39, pp. 1996-2003, 1998.

[12] H. du Raan, P. D. du Toit, A. van Aswegen, M. G. Lotter, C. P. Herbst, T. N. van der Walt, and A. C. Otto, "Implementation of a Tc-99 m and Ce-139 scanning line source for attenuation correction in SPECT using a dual opposing detector scintillation camera," Med. Phys., vol. 27, pp. $1523-1534,2000$.

[13] D. F. Yu, J. A. Fessler, and E. P. Ficaro, "Maximum-likelihood transmission image reconstruction for overlapping transmission beams," IEEE Trans. Med. Imag., vol. 19, no. 11, pp. 1094-1105, Nov. 2000.

[14] J. E. Bowsher, M. P. Tornai, J. Peter, D. E. González Trotter, A. Krol, D. R. Gilland, and R. J. Jaszczak, "Modeling the axial extension of a transmission line source within iterative reconstruction via multiple transmission sources," IEEE Trans. Med. Imag., vol. 21, no. 3, pp. 200-215, Mar. 2002.

[15] D. R. Gilland, R. J. Jaszczak, T. G. Turkington, and R. E. Coleman, "Comparison of transmission acquisition approaches for SPECT nonuniform attenuation compensation," IEEE Trans. Nucl. Sci., vol. 45, no. 3, pp. 1244-1249, Jun. 1998.

[16] M. P. Tornai, J. E. Bowsher, C. D. Stone, R. J. Jaszczak, and G. Enos, "Evaluation of transmission source collimation for SPECT attenuation compensation," in Conf. Rec. 1999 IEEE Nucl. Sci. Symp. Med. Imag. Conf., Seattle, WA, Oct. 24-30, 1999, pp. 1368-1372.

[17] S. H. Manglos, D. A. Bassano, and F. D. Thomas, "Cone-beam transmission computed tomography for nonuniform attenuation compensation of SPECT images," J. Nucl. Med., vol. 32, pp. 1813-1820, 1991. 
[18] Z. J. Cao and B. M. W. Tsui, "Performance characteristics of transmission imaging using a uniform sheet source with parallel-hole collimation," Med. Phys., vol. 19, pp. 1205-1212, 1992.

[19] P. Tan, D. L. Bailey, S. R. Meikle, S. Eberl, R. R. Fulton, and B. F. Hutton, "A scanning line source for simultaneous emission and transmission measurements in SPECT," J. Nucl. Med., vol. 34, pp. 1752-1760, 1993.

[20] B. J. Kemp, F. S. Prato, and R. L. Nicholson, "The geometric modulation transfer function of a transmission imaging system that uses a SPECT scintillation camera and parallel hole collimation," Med. Phys., vol. 26, pp. 733-741, 1995.

[21] J. M. Ollinger, "Maximum-likelihood reconstruction of transmission images in emission computed tomography via the EM algorithm," IEEE Trans. Med. Imag., vol. 21, no. 1, pp. 89-101, Mar. 1994.

[22] K. Lange and R. Carson, "EM reconstruction algorithms for emission and transmission tomography," J. Comput. Assist. Tomogr., vol. 8, pp. 306-316, 1984.

[23] H. Erdoğan and J. A. Fessler, "Monotonic algorithms for transmission tomography," IEEE Trans. Med. Imag., vol. 18, no. 9, pp. 801-814, Sep. 1999.
[24] — - "Ordered subsets algorithms for transmission tomography," Phys. Med. Biol., vol. 44, pp. 2835-2851, 1999.

[25] A. R. De Pierro, "On the relation between the ISRA and the EM algorithm for positron emission tomography," IEEE Trans. Med. Imag., vol. 12 , no. 2, pp. 328-333, Jun. 1993.

[26] K. Lange, Numerical Analysis for Statisticians. New York: SpringerVerlag, 1999.

[27] B. M. W. Tsui, X. D. Zhao, G. K. Gregoriou, D. S. Lalush, E. C. Frey, and R. E. Johnson, "Quantitative cardiac SPECT reconstruction with reduced image degradation due to patient anatomy," IEEE Trans. Nucl. Sci., vol. 41, no. 6, pp. 2838-2844, Dec. 1994.

[28] P. J. Huber, Robust Statistics. New York: Wiley, 1981.

[29] V. A. Brookeman and T. J. Bauer, "Collimator performance for scintillation camera systems," J. Nucl. Med., vol. 14, pp. 21-25, 1973.

[30] D. R. Gilland, B. M. W. Tsui, W. H. McCarthy, J. R. Perry, and J. Berg, "Determination of the optimum filter function for SPECT imaging," $J$. Nucl. Med., vol. 29, pp. 642-650, 1988.

[31] Z. Liang, J. Cheng, and J. Ye, "Validation of the central-ray approximation for attenuated depth-dependent convolution in quantitative SPECT reconstruction," Phys. Med. Biol., vol. 42, pp. 433-439, 1997. 